\title{
Malaria por Plasmodium vivax o P. falciparum en hospital de tercer nivel en la región más endémica de Colombia
}

\author{
Malaria due to Plasmodium vivax or P. falciparum \\ in a tertiary hospital in the most endemic region of \\ Colombia
}

\author{
Jaime Carmona-Fonseca • Medellín (Colombia), Yeliana lucía Sánchez, \\ María Fernanda Yasnot • Montería (Colombia)
}

\section{Resumen}

Problema: en Colombia hay pocos informes sobre paludismo grave, en especial producidos bajo protocolos bien sustentados.

Objetivo: describir las características clínicas de pacientes del Hospital San Jerónimo (HSJ), de Montería, en 2009-2010, e identificar y cuantificar los síndromes clínicos graves.

Metodología: estudio descriptivo, retrospectivo, transversal, de todos los pacientes hospitalizados con diagnóstico de paludismo.

Resultados: hubo 126 pacientes con paludismo en el HSJ; 74\% fueron remitidos de municipios circunvecinos; Plasmodium vivax causó $60 \%$ de los casos; $54 \%$ fueron mujeres (29 de 68 embarazadas, es decir, con malaria gestacional); fueron pacientes jóvenes (23.3 \pm 19.3 años); el promedio de evolución de su paludismo actual fue cuatro a cinco días; la parasitemia promedio al ingresar al HSJ fue $13628 \pm 26790$ parásitos/microlitro. La triada clásica de fiebre, escalofrío y cefalea se encuentra en al menos dos de cada tres pacientes al ingresar. El promedio de la hemoglobina indica anemia moderada. Los 126 pacientes aportaron 194 eventos de complicaciones. Las complicaciones más frecuentes fueron (porcentaje sobre 126 enfermos): trombocitopenia $76 \%$; anemia ( $<7 \mathrm{~g} / \mathrm{dL} \mathrm{de}$ hemoglobina) $17 \%$; falla renal $10 \%$; falla hepática $10 \%$; complicación cerebral (fiebre + convulsiones, sin coma: siete casos; fiebre + convulsiones + coma: un caso) $6 \%$; cinco casos fueron por $P$. vivax, dos por P. falciparum y uno por ambas especies (mixta); falla de dos a tres sistemas $11 \%$.

Conclusiones: se requiere adelantar estudios en Colombia sobre malaria grave que evalúen de manera integral los pacientes, con protocolos bien definidos, y que comparen criterios diagnósticos de gravedad y pautas de tratamiento para poder elaborar una norma autóctona sobre la materia. (Acta Med Colomb 2015; 40-294-304).

Palabras clave: malaria, paludismo, Plasmodium vivax, Plasmodium falciparum, Colombia .

\section{Abstract}

Problem: in Colombia there are few reports on severe malaria, especially produced under well supported protocols.

Objective: to describe the clinical characteristics of patients at the Hospital San Jeronimo (HSJ) of Montería, 2009-2010, and identify and quantify the severe clinical syndromes.

Methodology: a descriptive, retrospective, cross-sectional study of all patients hospitalized with a diagnosis of malaria.

Results: there were 126 patients with malaria in the HSJ; 74\% were referred from surrounding municipalities; Plasmodium vivax caused $60 \%$ of cases; $54 \%$ were women ( 29 of 68 were pregnant women, namely with gestational malaria); patients were young $(23.3 \pm 19.3$ years $)$; the average evolution of its current malaria was 4-5 days; the average parasitemia when entering the HSJ was $13628 \pm 26790$ parasites / microliter. The classic triad of fever, chills and headache were found in at least two out of three patients on admission. The average hemoglobin indicates moderate anemia.
Dr. Jaime Carmona-Fonseca: Grupo Salud y Comunidad-César Uribe Piedrahita, Universidad de Antioquia. Medellín; Dras. Yeliana Lucía Sánchez López y María Fernanda Yasnot A.: Grupo de Investigaciones Microbiológicas y Biomédicas de Córdoba (GIMBIC), Universidad de Córdoba. Montería (Colombia). Correspondencia. Dr. Jaime Carmona-Fonseca. Medellín (Colombia).

E-mail: jaimecarmonaf@hotmail.com Recibido: 12/XI/2014 Aprobado: 21/X/2015 
The 126 patients provided 194 adverse events and complications. The most frequent complications were (percentage of 126 patients): thrombocytopenia 76\%; anemia ( $<7 \mathrm{~g} / \mathrm{dL}$ hemoglobin) 17\%; renal failure $10 \%$; liver failure $10 \%$; cerebral complication (fever + seizures without coma: 7 cases, fever + convulsions + coma: 1 case) $6 \%$; five cases were due to P. vivax, 2 to P. falciparum and 1 to both species (mixed); failure of 2-3 systems $11 \%$.

Conclusions: the development of studies on severe malaria is required in Colombia to comprehensively assess patients with well-defined protocols and diagnostic criteria which compare diagnostic criteria of severity and treatment guidelines to develop an indigenous rule on the matter.

(Acta Med Colomb 2015; 40-294-304).

Keywords: malaria, paludism, Plasmodium vivax, Plasmodium falciparum, Colombia.

\section{Qué es malaria grave}

\section{Introducción}

La OMS dijo en 2000 que la "malaria grave es causada por Plasmodium falciparum" (1). Esa afirmación no es correcta ahora en 2014, porque ya se sabe que el paludismo grave es debido con más frecuencia a $P$. falciparum, pero que también $P$. vivax y $P$. knowlesi producen formas graves $y$ letales (2-9).

La OMS formuló en 1986 pautas para definir qué es malaria grave (severe malaria, en inglés) (10) y cuatro años después apareció una nueva y corregida versión (11). En 2000 apareció un tercer documento sobre el tema, referido exclusivamente a malaria grave por $P$. falciparum, sin referirse a P. vivax (12). La información disponible en 2000 llevó a los expertos de la OMS a modificar la definición de la malaria grave dada antes.

Todavía hoy, en 2015, no se tiene una pauta de la OMS sobre paludismo grave por P. vivax. En 2009, varios autores de India afirmaban (6) que la investigación sobre malaria por $P$. vivax es muy insuficiente porque se ha pensado que solo causa enfermedad benigna y sin complicaciones, pero sabemos ya que no es así (2-9).

Las manifestaciones graves informadas por monoinfección $P$. vivax son similares a las de infección grave por $P$. falciparum (6). El número de casos de paludismo por $P$. vivax está aumentando en todo el mundo, incluidos las formas graves. Parece que la infección con $P$. vivax induce una respuesta inmune inflamatoria más intensa que la inducida por P. falciparum (6, 9, 13-15). En América del Sur hay informes de paludismo grave por $P$. vivax, como anemia grave (16), de infección crónica en un paciente con esplenomegalia y trombocitopenia grave (7-8), de paludismo cerebral infantil (17), anemia y trombocitopenia graves e ictericia (3, 5). En Manaos (noroeste de la Amazonia de Brasil), se describieron los hallazgos en 17 pacientes con paludismo grave por $P$. vivax, confirmado por PCR: anemia grave cinco casos, ictericia diez, falla renal aguda dos, hemoglobinuria uno, síndrome de dificultad respiratoria aguda SDRA dos y choque un caso (2).

"Teniendo en cuenta los hallazgos clínicos y parasitológicos, la OMS considera como malaria complicada, independientemente de la especie causal, el cuadro clínico que se presenta con confusión, postración, malaria cerebral (definida como la presencia de coma no atribuible a otra causa), convulsiones generalizadas, anemia normocítica grave, hipoglicemia, acidosis metabólica con compromiso respiratorio, alteraciones hidroelectrolíticas, falla renal aguda, edema pulmonar agudo o síndrome de dificultad respiratoria del adulto, colapso circulatorio, choque, sangrado anormal, ictericia, hemoglobinuria, hiperpirexia o hiperparasitemia (para el caso de Colombia, por encima de 50000 formas asexuadas por $\mu \mathrm{L}$ ). Aunque en general la presencia de estos eventos es crucial para el diagnóstico de malaria complicada, existen grandes diferencias entre la forma de presentación de la malaria en adultos cuando se la compara con la de niños menores de cinco años, siendo más frecuente el compromiso neurológico y respiratorio en los niños y el hepático en los adultos" (14).

\section{Frecuencia del paludismo en Colombia}

El paludismo presenta frecuencia alta y decreciente en los últimos diez años en Colombia, tendencia vista en general en el mundo, por razones no claras. En 2003-2013 los casos variaron entre 59222 (2013) y 154077 (2003) y se esperan alrededor de 40000 en 2014 (datos del Instituto Nacional de Salud: Programa Sivigila). El promedio aritmético de 2003-2013 es 120812 casos/año y la mediana es 113137 (cálculos nuestros con los datos oficiales).

Los casos de paludismo en el departamento de Córdoba en 2001-2012 fueron, en promedio, 26 000/año, a lo que corresponde un índice parasitario anual (IPA) de 16.23 por mil expuestos. La tendencia es francamente decreciente (Tabla 1; Figura 1) (18). Los cuatro municipios de mayor endemia en todo ese periodo fueron Tierralta, Puerto Libertador, Montelíbano y Valencia, en orden decreciente. Los valores de IPA en 2010-2012 para esos cuatro municipios aparecen en la Tabla 2 (18). La Figura 2 corresponde al mapa de riesgo malárico para los municipios de Córdoba en 2007 (19).

Los informes sobre paludismo grave o complicado son escasos en Colombia. El presente documento trata sobre un estudio descriptivo retrospectivo adelantado en el Hospital San Jerónimo de Montería, capital del departamento de Córdoba, en el noroeste colombiano, en la zona que más paludismo genera en el país (20-22). Estos pacientes fueron hospitalizados por disposición médica, sin atenerse a ningún 
Tabla 1. Malaria en Córdoba y los cuatro municipios más endémicos, 2001-2012.

\begin{tabular}{|l|c|c|c|c|c|}
\hline Año & Córdoba & Montelibano & P. Libertador & Tierralta & Valencia \\
\hline 2001 & 45316 & 12342 & 9766 & 15213 & 6982 \\
2002 & 38152 & 10354 & 10004 & 13233 & 4380 \\
2003 & 35822 & 8500 & 9794 & 13218 & 3836 \\
2004 & 30537 & 4695 & 6054 & 16494 & 2625 \\
2005 & 24025 & 3571 & 7070 & 10404 & 2860 \\
2006 & 24906 & 2729 & 10543 & 7608 & 2960 \\
2007 & 46197 & 7807 & 17801 & 15608 & 3116 \\
2008 & 17760 & 3805 & 4856 & 5040 & 3109 \\
2009 & 16644 & 3588 & 4120 & 4414 & 3869 \\
2010 & 18233 & 2703 & 7097 & 5666 & 1961 \\
2011 & 9458 & 2401 & 2416 & 3162 & 637 \\
2012 & 4530 & 1397 & 1408 & 1791 & 128 \\
\hline media & $\mathbf{2 5 9 6 5}$ & $\mathbf{5 3 2 4}$ & $\mathbf{7 5 7 7}$ & $\mathbf{9 3 2 1}$ & $\mathbf{3 0 3 9}$ \\
\hline Fuente: datos obtenidos en la Secretaría Departamental de Salud de Córdoba. \\
\hline
\end{tabular}

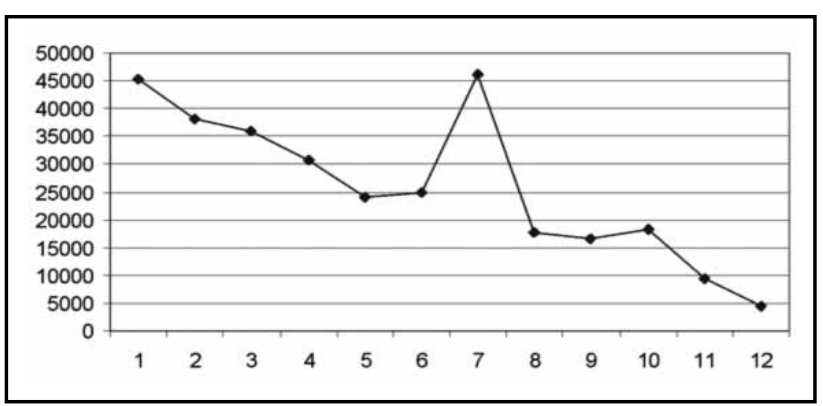

Figura 1. Malaria en Córdoba, 2001-2012: casos por año. Fuente: elaboración nuestra con datos obtenidos en la Secretaría Departamental de Salud de Córdoba. criterio de gravedad de la OMS o de otra índole; sólo intervino la opinión del médico tratante. Los objetivos de esta revisión de casos clínicos hospitalarios fueron: a) Medir la incidencia de malaria y medir la mortalidad por paludismo entre los pacientes hospitalizados en el referido hospital; b) Hacer un inventario de los cuadros o síndromes clínicos hallados; c) Explorar la calidad del tratamiento antipalúdico específico recibido por los pacientes.

\section{Metodología}

El sitio del estudio: Hospital San Jerónimo de Montería, departamento de Córdoba.

El departamento de Córdoba, en Colombia, ocupa una zona en la parte noroccidental del país, con costas sobre el mar Caribe (Figura 2). Tiene extensión aproximada de $24 \mathrm{~km}^{2}, 1600000$ habitantes, todos expuestos al riesgo de

Tabla 2. Malaria en los cuatro municipios del sur de Córdoba, Colombia, con más endemia de paludismo; 2010-2012.

\begin{tabular}{|c|c|c|c|c|c|c|c|c|c|}
\hline \multirow[t]{2}{*}{ Municipio } & \multicolumn{3}{|c|}{2010} & \multicolumn{3}{|c|}{2011} & \multicolumn{3}{|c|}{2012} \\
\hline & casos & IPA & $\mathbf{N}$ & casos & IPA & $\mathbf{N}$ & casos & IPA & $\mathbf{N}$ \\
\hline Tierralta & 6255 & 70.6 & 88582 & 3162 & 34.8 & 90738 & 1791 & 19.3 & 92948 \\
\hline P Libertador & 7177 & 176.8 & 40584 & 2416 & 57.6 & 41924 & 1408 & 32.5 & 43287 \\
\hline Montelibano & 3094 & 42.6 & 72565 & 2401 & 32.3 & 74284 & 1397 & 18.4 & 76010 \\
\hline Valencia & 2159 & 56.2 & 38387 & 637 & 16.2 & 39258 & 128 & 3.2 & 40160 \\
\hline Total & 18685 & 77.8 & 240118 & 8616 & 35.0 & 246204 & 4724 & 18.7 & 252405 \\
\hline
\end{tabular}

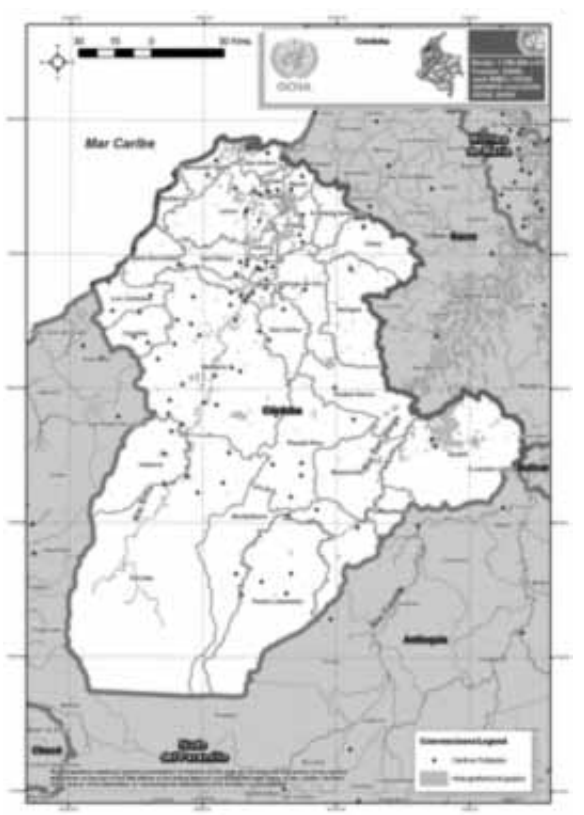

Fuente: http://www.colombiassh.org/site/IMG/png/Cordoba_A3_sin.png

Figura 2. Mapa de riesgo de malaria en Córdoba, 2007. Al centro y norte, zona plana de pastizales y agroindustria. Baja-media endemia de malaria. 
paludismo. El territorio cordobés está dividido en 30 municipios, cinco de los cuales pertenecen a la zona costanera, 16 a la zona o cuenca del Sinú, y nueve a la del San Jorge (23).

Montería es la capital del departamento de Córdoba y tiene aproximadamente 500000 habitantes. El Hospital San Jerónimo de Montería (HSJ) es el único hospital público de esa ciudad y es el único centro de niveles II y III del departamento. A él acuden mayoritariamente personas de estrato socioeconómico bajo (niveles 1-3 del sistema colombiano de clasificación socioeconómica: Sisben).

\section{Tipo de estudio, diseño y tamaño muestrales}

Es un estudio descriptivo, retrospectivo y transversal, con información secundaria. Se consultó el registro de egresos hospitalarios del HSJ entre enero de 2009 y junio de 2010 para conocer los números de historias clínicas de pacientes con diagnóstico de egreso de malaria, solo o asociado a otros términos (malaria, grave, malaria complicada, malaria cerebral, etc.). En el archivo de historias clínicas del HSJ se consultaron los registros para obtener los datos previstos sobre cada paciente hospitalizado. Se decidió trabajar con un tamaño muestral correspondiente a la totalidad de historias clínicas que cumpliesen con los criterios de inclusión.

\section{Criterios de inclusión}

Se revisaron todas las historias clínicas con diagnóstico de egreso de malaria, en el lapso referido. Se incluyeron en el estudio las historias que cumplieron estos criterios: a) suministrar los datos de sexo y edad del paciente; b) indicar el sitio de residencia del paciente; c) indicar el resultado del examen de gota gruesa que informaba sobre la especie de Plasmodium; d) contener la información sobre síntomas y signos del paciente en el momento del ingreso al HSJ; e) indicar el resultado de los exámenes de laboratorio practicados al paciente; f) expresar la situación final del paciente: egreso vivo o muerto; egreso por alta o por traslado.

\section{Definiciones de síndromes palúdicos}

Los síndromes palúdicos que pueden establecerse con base en las manifestaciones clínicas (síntomas, signos, resultados de laboratorio) son los siguientes, con los criterios de González et al 2000 (5) modificados levemente por nosotros, así:

Compromiso cerebral: si el paciente tiene simultáneamente fiebre y convulsiones, o fiebre y coma, o fiebre, convulsiones y coma. Con los tres criterios actuando en forma concomitante el diagnóstico de malaria cerebral parece altamente probable, haciéndose casi seguro si la especie parasitaria asociada es $P$. falciparum o éste y $P$. vivax en forma simultánea (malaria mixta).

Compromiso renal: el diagnóstico de falla o insuficiencia renal debe sospecharse claramente si hay concentración de creatinina sérica superior a $1 \mathrm{mg} / \mathrm{dL}$, así como con la presencia de oliguria, de anuria, de poliuria o de varias de ellas. Si los dos criterios se consideran en forma concomi- tante (creatinina alta y trastorno de la eliminación urinaria), el diagnóstico de complicación o compromiso renal es casi seguro.

Síndrome renal 1: creatinina $>1 \mathrm{mg} / \mathrm{dL}$ o presencia de oliguria, de anuria, de poliuria o de varias de ellas.

Síndrome renal 2: creatinina $>1 \mathrm{mg} / \mathrm{dL}$ más presencia de oliguria, de anuria, de poliuria o de varias de ellas.

Compromiso hepático: el incremento de la bilirrubina indirecta (BI) en la sangre por arriba de $1 \mathrm{mg} / \mathrm{dL}$, o de alguna de las transaminasas: aspartato amino tranferasa (ASP) (antes GOT) mayor de 35 UI, o alanino amino transferasa (ALT) (antes GPT) más de 40 UI, hace posible el diagnóstico de compromiso hepático. Si consideramos en forma simultánea la alteración de las tres variables (BI, ASP/GOT y ALT/ GPT), el compromiso hepático es casi seguro, pudiéndose afirmar su presencia indiscutible si, además, existe ictericia. Síndrome hepático 1: $\mathrm{BI}>1,0$; Síndrome hepático 2: $\mathrm{BI}>$ $1,0+$ GOT $>35+$ ALT $>40$.

Compromiso tipo edema pulmonar: el diagnóstico parece garantizado si existen disnea y rayos $\mathrm{X}$ de pulmón con hallazgos compatibles con esta complicación.

Anemia grave: valor de hemoglobina inferior a: grado 1: $<7 \mathrm{~g} / \mathrm{dL}$ : es suficiente para hacer el diagnóstico; grado 2: $<5 \mathrm{~g} / \mathrm{dL}$ : es suficiente para hacer el diagnóstico.

Trombocitopenia: trombocitopenia-1: recuento inferior a $150000 \times 10^{6}$ plaquetas $/ \mathrm{mL}$; trombocitopenia-2: recuento inferior a $50000 \times 10^{6}$ plaquetas $/ \mathrm{mL}$.

Hipoglicemia grave: se usan dos puntos de corte (umbrales) para la glicemia, así: menos de $40 \mathrm{mg} / \mathrm{dL}$, que es suficiente para formular el diagnóstico de esta complicación, y menos de $61 \mathrm{mg} / \mathrm{dL}$, considerándose que entre 40 y 60 $\mathrm{mg} / \mathrm{dL}$ es muy probable que existan manifestaciones clínicas debidas a hipoglicemia. Se usaron, entonces, dos puntos de corte (umbrales diagnósticos): $<61$ y $<40 \mathrm{mg} / \mathrm{dL}$.

Compromiso multisistémico: presencia de dos o más de los anteriores daños.

\section{Análisis estadístico}

Los datos se analizaron con el programa SPSS 10.0. Se calcularon medidas de tendencia central y de dispersión, así como proporciones. Se compararon medidas de tendencia central de variables métricas con la prueba no paramétrica de Kruskal-Wallis y se compararon distribuciones de frecuencias de variables no métricas con la prueba ji al cuadrado de Pearson. Se midieron correlaciones lineales bivariadas con la prueba no paramétrica para el rho de Spearman.

Todas las decisiones sobre significación se tomaron con una probabilidad (p) menor de 0.05 .

\section{Aspectos éticos}

Las historias clínicas y los datos obtenidos se manejaron exclusivamente por los investigadores y cada historia se registró sólo con códigos numéricos, nunca con el nombre de los pacientes. Las autoridades del HSJ y el Comité de Ética del Instituto de Investigaciones Médicas de la Facultad 
de Medicina de la Universidad de Antioquia aprobaron el proyecto de investigación en cuanto a los aspectos éticos.

\section{Resultados}

Se hallaron 126 pacientes hospitalizados en el HSJ en ese lapso de 18 meses, a quienes el diagnóstico principal o primero en formularse fue "malaria", a veces sin más términos acompañantes, otras acompañado de la expresión "grave", otras veces manifestada como "malaria cerebral", entre otras expresiones. Un total de $26 \%$ (33/126) de los pacientes llegaron directamente al HSJ y 74\% (93/126) fueron remitidos de otras instituciones de salud: 5\% de municipios del vecino departamento de Antioquia y $69 \%$ de municipios de Córdoba (Tabla 3).

Especie plasmodial: la especie informada por la gota gruesa practicada en el HSJ fue $P$. vivax $60 \%$ (76/126), $P$. falciparum $31 \%$ (39/126) y ambas especies (malaria mixta) $9 \%$ (11/126). La parasitemia, en el HSJ, se hizo sólo a $41 \%(52 / 126)$ de los pacientes. La parasitemia varió entre 3 y 130 000/uL. En 10\% (5/54) de los casos la parasitemia fue $>50000 / \mathrm{uL}$. Los valores centrales de la parasitemia fueron $13628 \pm 26790$ y los percentiles 25, 50 y 75 fueron 1292, 3096 y 10 835. La distribución de la parasitemia fue fuertemente asimétrica, con cola derecha. La parasitemia de P. vivax (14 casos) fue de 7534 \pm 8468 (entre 900 y $33600 / \mathrm{uL}$ ) y la de $P$. falciparum de $18514 \pm 33525$ (entre 3 y 130000 ). En paludismo mixto, la parasitemia fue $4179 \pm 3381 / \mathrm{uL}$ (entre 900 y 9680).

Tabla 3. Origen de los pacientes hospitalizados con malaria en el HSJ, Montería, 2009-2010.

\begin{tabular}{|c|c|c|c|}
\hline Remitido de: & Municipio & $\mathbf{n}$ & $\%$ \\
\hline NO remitidos & & 33 & 26.2 \\
\hline De Antioquia & & 6 & 4.8 \\
\hline \multirow[t]{2}{*}{ Urabá } & Arboletes & 2 & 1.6 \\
\hline & Necoclí & 1 & 0.8 \\
\hline \multirow[t]{3}{*}{ Bajo Cauca } & Caucasia & 1 & 0.8 \\
\hline & El Bagre & 1 & 0.8 \\
\hline & Nechi & 1 & 0.8 \\
\hline \multirow[t]{10}{*}{ De Córdoba } & & 87 & 69.0 \\
\hline & Tierralta & 35 & 27.8 \\
\hline & P. Libertador & 11 & 8.7 \\
\hline & Valencia & 9 & 7.1 \\
\hline & Montelíbano & 9 & 7.1 \\
\hline & Planeta Rica & 8 & 6.3 \\
\hline & Buenavista & 4 & 3.2 \\
\hline & Ayapel & 3 & 2.4 \\
\hline & San Pelayo & 2 & 1.6 \\
\hline & Otros seis municipios a & 6 & 4.8 \\
\hline \multicolumn{2}{|l|}{ Total } & 126 & 100. \\
\hline $\begin{array}{l}\text { a Un paciente de cad } \\
\text { dido, Pueblo Nuevo, }\end{array}$ & $\begin{array}{l}\text { de estos lugares: Can } \\
\text { gún. }\end{array}$ & & rto $\mathrm{E}$ \\
\hline
\end{tabular}

Carácterísticas epidemiológicas generales: las características generales de los 126 pacientes al ingresar al HSJ aparecen en la Tabla 4-A. Las mujeres fueron mayoría, con $54 \%$ de los pacientes. La edad varió de menos de uno a 89 años (<1 año: tres niños; 1-4: 13; 5-9: 14; 10-14: nueve; subtotal 0-14 años hubo 43 pacientes; $15-17$ : 11; 18-25: 27; 26-50: $30 ; \geq 51$ : 15 casos).

Tabla 4. Hallazgos clínicos en el ingreso al HSJ; Montería, 2009-2010.

\begin{tabular}{|c|c|c|c|c|c|}
\hline \multicolumn{6}{|l|}{ A. } \\
\hline Característica & $\mathbf{N}$ & Mínimo & Máximo & Media & Desv. típ. \\
\hline Edad & 126 & 0 & 89 & 23.30 & 19.29 \\
\hline Días de evolución a & 126 & 0 & 19 & 4.68 & 3.15 \\
\hline PAS mm Hg & 110 & 70 & 140 & 10.51 & 1.46 \\
\hline PAD mm $\mathrm{Hg}$ & 110 & 40 & 100 & 6.66 & 1.15 \\
\hline $\mathrm{FC} / \mathrm{min}$ & 124 & 20 & 140 & 89.40 & 16.58 \\
\hline $\mathrm{FR} / \mathrm{min}$ & 126 & 16 & 81 & 24.16 & 9.82 \\
\hline Temperatura ${ }^{\circ} \mathrm{C}$ & 124 & 36 & 41 & 37.514 & 1.004 \\
\hline \multicolumn{6}{|c|}{$\begin{array}{l}\text { a Días de evolución del paludismo actual; Presiones arterial sistólica PAS y diastólica } \\
\text { PAD; frecuencias cardiaca FC y respiratoria FR. }\end{array}$} \\
\hline \multicolumn{6}{|l|}{ B. } \\
\hline \multicolumn{3}{|c|}{ Síntoma o signo (si, no) } & \multicolumn{2}{|l|}{$\mathbf{n}$} & $\%$ \\
\hline \multicolumn{3}{|l|}{ Fiebre } & \multicolumn{2}{|l|}{120} & 95.2 \\
\hline \multicolumn{3}{|l|}{ Escalofrío } & \multicolumn{2}{|l|}{76} & 70.3 \\
\hline \multicolumn{3}{|l|}{ Cefalea } & \multicolumn{2}{|l|}{82} & 65.1 \\
\hline \multicolumn{3}{|l|}{ Mareo } & \multicolumn{2}{|l|}{26} & 20.6 \\
\hline \multicolumn{3}{|l|}{ Vómito } & \multicolumn{2}{|l|}{51} & 40.5 \\
\hline Diarrea & & & 14 & & 11.1 \\
\hline Tos & & & 13 & & 10.3 \\
\hline Debilidad & & & 28 & & 22.2 \\
\hline Adinamia & & & 26 & & 20.6 \\
\hline Astenia & & & 24 & & 19.2 \\
\hline Deshidratación & & & 9 & & 7.2 \\
\hline Hiperestesia zona he & tica & & 3 & & 2.4 \\
\hline Hepatomegalia & & & 20 & & 16.0 \\
\hline Ictericia & & & 26 & & 20.6 \\
\hline Coluria & & & 8 & & 6.3 \\
\hline Somnolencia & & & 12 & & 9.5 \\
\hline Obnubilación & & & 2 & & 1.6 \\
\hline Convulsiones & & & 7 & & 5.6 \\
\hline Rigidez nuca & & & 0 & & 0 \\
\hline Coma & & & 1 & & 0.8 \\
\hline Confusión & & & 1 & & 0.8 \\
\hline Reflejo pupilar & & & 8 & & 6.3 \\
\hline Estertores & & & 17 & & 13.5 \\
\hline Roncos & & & 7 & & 5.6 \\
\hline Sibilancias & & & 3 & & 2.4 \\
\hline Hemoptisis & & & 1 & & 0.8 \\
\hline Taquipnea & & & 6 & & 4.8 \\
\hline Taquicardia & & & 4 & & 3.2 \\
\hline Hipovolemia & & & 1 & & 0.8 \\
\hline Oliguria & & & 3 & & 2.4 \\
\hline Anuria & & & 1 & & 0.8 \\
\hline Poliuria & & & 1 & & 0.8 \\
\hline Edema & & & 7 & & 5.6 \\
\hline Petequias & & & 4 & & 3.2 \\
\hline Otros & & & 17 & & 13.5 \\
\hline
\end{tabular}


Entre las 68 mujeres, $23 \%$ (29/126) estaban en embarazo; entre las gestantes se halló: la edad fue de 16-32 años (media: 22); los días de evolución del paludismo actual, en el momento de la consulta, fueron dos en tres mujeres y una en las otras 26.

El antecedente de haber tenido malaria en algún momento de la vida fue narrado por $26 \%$ (32/125) pacientes, que en promedio tuvieron 1.40 ataques previos. Solo 18 de 32 pacientes informaron sobre cuándo había ocurrido tal episodio y el mismo sucedió entre tres y 3650 días (10 años) antes del actual, con promedio de 525 días (1.44 años).

Los pacientes fueron jóvenes (edad promedio: 23 años). Los días de evolución del actual episodio de paludismo variaron entre menos de uno y 19 días; únicamente $12 \%$ de los pacientes consultaron antes, de dos y $32 \%$ lo hicieron entre dos y tres días; $30 \%$ demoraron seis a 19 días; el promedio de evolución fue cuatro a cinco días. La demora para la consulta luego de instalado el ataque palúdico fue de cero a cinco días en 71\% (89/126) de los pacientes. Los días de evolución fueron similares para pacientes remitidos y no remitidos al HSJ ( $p=291)$, así como para los remitidos de municipios de Antioquia o de Córdoba ( $\mathrm{p}=0.316$ ).

Aspectos clínicos: el valor promedio de los signos vitales estuvo en el intervalo fisiológico. Los síntomas y signos clínicos presentes en el ingreso al HSJ se presentan en la Tabla 4-B. La triada clásica de fiebre, escalofrío y cefalea se encuentra en al menos dos de cada tres pacientes, en el momento del ingreso al HSJ. Se destacan las frecuencias de vómito (40\%), diarrea (11\%) y tos (10\%). También son notorias las frecuencias de hepatomegalia (16\%), ictericia $(21 \%)$, estertores pulmonares (13\%). Llama mucho la atención, por su muy escasa frecuencia, la palidez.

Los resultados del hemograma y las pruebas de laboratorio clínico practicadas al ingresar el paciente al hospital están en la Tabla 5. La cantidad de pacientes con evaluación completa de laboratorio es muy baja. Solo el hemograma se hizo,

Tabla 5. Resultados de pruebas de laboratorio clínico: variable y valores de referencia.

\begin{tabular}{|c|c|c|c|c|c|c|}
\hline \multicolumn{2}{|l|}{ Serie Roja $n=125$} & \multirow{2}{*}{$\begin{array}{l}\text { Min } \\
1.08\end{array}$} & \multirow{2}{*}{$\begin{array}{c}\text { Max } \\
37.0\end{array}$} & \multirow{2}{*}{$\begin{array}{c}\text { Media } \\
3.5794\end{array}$} & \multirow{2}{*}{$\begin{array}{c}\text { Desv est } \\
3.1280\end{array}$} & \multirow{2}{*}{$\frac{\text { Nota }}{\text { Bajos }}$} \\
\hline Eritrocitos & 3.8-6.0 millones/uL & & & & & \\
\hline Hemoglobina & $\begin{array}{l}\geq 11 \text { g/dL } 0.5-6 \text { años; } \\
\geq 12 \text { g/dL } 7 \text { y más años }\end{array}$ & 3.5 & 14.1 & 9.100 & 2.386 & Anemia en promedio \\
\hline Hematocrito $\mathrm{Hb} \times 3$ & & 8.6 & 52.1 & 26.990 & 7.191 & Bajo en promedio \\
\hline $\mathrm{VCM}^{\mathrm{a}}$ & $80-100 \mathrm{fL}$ & 58.7 & 126.7 & 81.440 & 8.944 & \multirow{3}{*}{ En límite inferior normal } \\
\hline HCM & $27-31 \mathrm{picog} / \mathrm{cel}$ & 18.3 & 87.1 & 27.872 & 6.806 & \\
\hline $\mathrm{CHCM}$ & $32-36 \mathrm{~g} / \mathrm{dL}$ & 28.2 & 40.4 & 33.722 & 3.433 & \\
\hline $\mathrm{ADE}$ & $11.5-14.5 \%$ & 10.5 & 35.3 & 16.095 & 3.524 & Mayor que límite superior normal \\
\hline \multicolumn{7}{|c|}{ a VCH volumen corpuscular medio; HCM hemoglobina corpuscular media; CGCM concentración de HCM; ADE ancho de distribución eritrocitaria. } \\
\hline Plaquetas $n=125$ & & Min & Max & Media & Desv est & Nota \\
\hline $150000-450000 / \mathrm{uL}$ & & 12000 & 568000 & 110000 & 100000 & Trombocitopenia \\
\hline Serie Blanca $\mathrm{n}=125$ & & Min & Max & Media & Desv est & Nota \\
\hline Leucocitos & $4800-10000$ & 1300 & 22100 & 7528 & 3545 & \multirow{6}{*}{ Normal } \\
\hline Granulocitos (\%) & $44 \%-74$ & & & & & \\
\hline Neutrófilos (\%) & $45-75$ & 5.3 & 95.2 & 59.9 & 19.7 & \\
\hline Eosinófilos (\%) & $1-5$ & 0.0 & 15.0 & 1.7 & 2.4 & \\
\hline Basófilos (\%) & $0-2$ & 0.0 & 1.5 & 0.35 & 0.33 & \\
\hline Linfocitos $(\%)$ & $21-45$ & 4.1 & 92.2 & 28.5 & 18.1 & \\
\hline Monocitos (\%) & 4-9 & 0.0 & 35.9 & 9.4 & 5.7 & En límite superior normal \\
\hline \multicolumn{2}{|c|}{ Química sanguínea casos; valores de referencia } & Min & Max & Media & D.E & Nota \\
\hline Glicemia $n=47$ & $70-100 \mathrm{mg} / \mathrm{dL}$ & 20 & 432 & 72.3 & 76 & Media normal; <60: 20.41. 56; >110: 11; 114 a 432 \\
\hline GOT/AST n= 37 & $\leq 35 \mathrm{UI} / \mathrm{L}$ & 8 & 140 & 43.7 & 28 & $>35: 17 ; 38$ a 140 \\
\hline GPT/ALT $n=36$ & $\leq 40 \mathrm{UI} / \mathrm{L}$ & 7 & 105 & 31.7 & 24 & $>40: 9 ; 44$ a 105 (todos GOT >35) \\
\hline $\mathrm{LDH}^{\mathrm{a}}$ plasma $\mathrm{n}=11$ & 190-390 UI/L & 483 & 2206 & 1.171 & 676 & $>390: 11 ; 5$ con GOT $>35$ y 1 ALT $>40$ \\
\hline Fosfatasas alcalinas $n=4$ & $40-190 \mathrm{U} / \mathrm{L}$ & 106 & 222 & 149.7 & 52 & $>190: 1$ \\
\hline Ácido úrico $\mathrm{n}=5$ & $2.5-7.2 \mathrm{mg} / \mathrm{dL}$ & 2.7 & 6.5 & 4.4 & 1.4 & Todos $<6.56$ \\
\hline Amilasa $\mathrm{n}=2$ & $23-85 \mathrm{U} / \mathrm{L}$ & 44 & 64 & 54.0 & 14.1 & Solo dos mediciones: 44 y 64 UI \\
\hline Creatinina $\mathrm{n}=61$ & $\mathrm{mg} / \mathrm{dL}$ 0.6-1.1 mujer; 0.8-1.3 hombre & 0.3 & 9.0 & 1.0 & 1.1 & Media normal; $\leq 1.20: 50 ;>1.20: 11$ (1.23 a 9.04) \\
\hline Úrea $n=31$ & $7-20 \mathrm{mg} / \mathrm{dL}$ & 2.9 & 268 & 46.9 & 48.4 & Media alta; > 20: 20 (24-268); 8 con creati-nina $>1.20$ \\
\hline $\mathrm{BUN}^{\mathrm{a}} \mathrm{n}=40$ & $7-21 \mathrm{mg} / 100 \mathrm{~mL}$ & 296 & 125 & 18.6 & 20.3 & Media normal; >21: 9; (31.8 a 125) \\
\hline $\mathrm{BT}^{\mathrm{a}} \mathrm{n}=34$ & $0.2-1.0 \mathrm{mg} / \mathrm{dL}$ & 0.5 & 34.0 & 4.0 & 6.2 & Media alta \\
\hline $\mathrm{BI} n=33$ & $0.1-0.5 \mathrm{mg} / \mathrm{dL}$ & 0.2 & 12.4 & 1.3 & 2.1 & $>1: 9(1.22-12.41)$. con BT 1.9-20.4 \\
\hline $\mathrm{BD} n=33$ & $0.3 \mathrm{mg} / \mathrm{dL}$ & 0.2 & 31.5 & 2.6 & 5.5 & Media alta \\
\hline $\mathrm{TP}^{\mathrm{a}} \mathrm{n}=21$ & $7-15$ seg & 3.6 & 70.0 & 21.0 & 12.1 & Alza leve \\
\hline TPT $n=21$ & $20-40 \mathrm{seg}$ & 25.0 & 40.1 & 32.2 & 5.1 & Normal; hacia límite superior \\
\hline
\end{tabular}


en general, a todos. Las transaminasas se hicieron a $29 \%$ de los casos; la creatinina a $48 \%$, urea a $25 \%$; bilirrubinas a $34 \%$; glicemia a $25 \%$; otras pruebas a escasos pacientes.

El promedio de la hemoglobina indica anemia moderada; $17 \%$ de 125 pacientes tiene hemoglobina $<7 \mathrm{~g} / \mathrm{dL}$ y $5 \%$ la tienen por debajo de $5 \mathrm{~g} / \mathrm{dL}$. El promedio de plaquetas $(100000 / \mathrm{uL})$ indica trombocitopenia. En general, los leucocitos tienen cantidad y distribución relativa normal. Los promedios de glicemia, creatinina y BUN son normales y los de urea y bilirrubinas son altos.

Para tratar de indagar sobre el origen del aumento de las bilirrubinas, se buscó la correlación entre cada una de ellas y las transaminasas. No hubo correlación de la bilirrubina indirecta con ninguna de las dos transaminasas - esto sugiere que el origen no es hemólisis-, pero la directa sí se correlacionó con ambas -sugiere que se debe a disfunción del hepatocito $-($ GOT rho $=0.543 ; \mathrm{p}=0.003 ; \mathrm{n}=27$; GPT rho= $0.535 ; \mathrm{p}=0.005 ; \mathrm{n}=26)$. lo mismo que la total (GOT rho= $0.403 ; \mathrm{p}=0.037 ; \mathrm{n}=27$; GPT $\mathrm{rho}=0.439 ; \mathrm{p}=0.025 ; \mathrm{n}=26$ ).

No hubo correlación de la bilirrubina indirecta con ninguna de las dos transaminasas, pero la directa sí se correlacionó con ambas $($ GOT rho $=0.543 ; \mathrm{p}=0.003 ; \mathrm{n}=27$; GPT rho=
$0.535 ; \mathrm{p}=0.005 ; \mathrm{n}=26)$. lo mismo que la total $(\mathrm{GOT}$ rho $=$ $0.403 ; \mathrm{p}=0.037 ; \mathrm{n}=27$; GPT $\mathrm{rho}=0.439 ; \mathrm{p}=0.025 ; \mathrm{n}=26$ ).

Síndromes clínicos: con los criterios de González et al 2000 , ligeramente modificados por nosotros, se estableció la presencia de varios síndromes clínicos asociados al actual ataque de malaria (Tabla 6). Entre los 126 pacientes aportaron 194 eventos de síndrome clínico de complicaciones (1,5 complicaciones por paciente). Quince pacientes tuvieron dos o tres síndromes (falla multisistémica).

Trombocitopenia: fue la complicación más frecuente; fue "leve" (<150 000/uL) en 76\% (96/126), "grave" $(<50$ 000/uL) en $29 \%$ (36/126) y "muy grave" (<20 000/uL) en $6 \%$. La ocurrencia de ninguno de estos síndromes se asoció estadísticamente con la especie de plasmodio.

Compromiso cerebral: sucedió en ocho pacientes: siete con fiebre y convulsiones; uno con fiebre y coma; ninguno con fiebre, convulsiones y coma. Ninguno de tres con glicemia $<60$ tuvo compromiso cerebral. Cinco casos fueron por $P$. vivax, dos por P. falciparum y uno por ambas especies (mixta). Seis casos fueron hombres y uno fue mujer. Tres de los siete fueron menores de 15 años (dos de cinco años y uno de 14), todos hombres, dos por $P$. vivax y uno por $P$.

Tabla 6. Síndromes clínicos asociados al ataque de paludismo en los 126 pacientes.

\begin{tabular}{|c|c|c|c|}
\hline Síndrome y criterios diagnósticos & $\mathbf{N}$ a(síndromes) & $\%^{a}(n=126$ pac. $)$ & Nota \\
\hline \multicolumn{4}{|l|}{ Una complicación } \\
\hline $\begin{array}{l}\text { Cerebral } \\
\text { simultáneamente fiebre y convulsiones, } \\
\text { o fiebre y coma, o fiebre, convulsiones y coma }\end{array}$ & 8 & 6 & $\begin{array}{l}\text { Fiebre y convulsiones: } 7 \\
\text { Fiebre y coma: } 1 \\
\text { Fiebre, convulsiones y coma: } 0 \\
\text { Ninguno de } 3 \text { con glicemia }<60 \text { tuvo compromiso cerebral. }\end{array}$ \\
\hline Renal-1 creatinina $>1,2$ & 10 & 8 & $\begin{array}{l}\text { Creatinina } 1.23-9.04 ; \text { sin oliguria ni anuria; } 2 \text { con edema; } 3 \text { con edema. } \\
\text { Todos con urea }>20 \mathrm{mg} / \mathrm{dL} \text { ( } 42 \text { a } 268 \text {. En } 4 \text { se midieron transaminasas } \\
\text { y } 4 \text { tuvieron GOT }>35 \text { pero sólo } 1 \text { ALT }>40 \text {. }\end{array}$ \\
\hline Renal-2 creatinina $>1.2+$ oliguria o anuria & 1 & $<1$ & $\begin{array}{l}\text { Hombre, } 30 \text { años, P. vivax; creatinina 9.04; con oliguria, anuria, edema; BI } 0.20 \text {, } \\
\text { BT 31.7; GOT/AST 60, GPT/ALT 22, LDG 1750; fosfatasas alcalinas } 150 .\end{array}$ \\
\hline Hepático-1 $\mathrm{BI}>1.0 \mathrm{mg} / \mathrm{dL}$ & 10 & 8 & $\begin{array}{l}4 \text { con GOT/AST }>35 ; 2 / 4 \text { con GPT/ALT }>40^{\text {b }} \\
4 \text { con creatinina }>1.0 \mathrm{y}\end{array}$ \\
\hline Hepático-2 BI $>1.0+\mathrm{GOT}>35+$ ALT $>40$ & 2 & $<1$ & c \\
\hline Edema pulmonar & 0 & 0 & Ninguno de los pacientes tiene Rx tórax. \\
\hline Anemia grave- $1<7 \mathrm{mg} / \mathrm{dL}$ & 21 & 17 & 5.0 a $6.9 ; 13 / 21$ con trombocitopenia. \\
\hline Anemia grave- $2<5 \mathrm{mg} / \mathrm{dL}$ & 6 & 0.5 & 3.5 a $4.9 ; 5 / 6$ con trombocitopenia. \\
\hline Trombocitopenia-1 plaquetas $<150000$ & 96 & 76 & Media: $65000 ; 85 \%$ con anemia \\
\hline Trombocitopenia- 2 plaquetas $<50000$ & 36 & 29 & Media: $30000 ; 86 \%$ con anemia. \\
\hline Hipoglicemia-1 <60 mg/dL (n=32) & 3 & 10 & Media: $106 ; 13$ con $<80$ y $11 \mathrm{cpn}>110$. \\
\hline Hipoglicemia-2 $<40$ mg/dL ( $\mathrm{n}=32$ ) & 1 & 3 & \\
\hline \multicolumn{4}{|l|}{ Varias complicaciones } \\
\hline Multisistémico: 2 sistemas & 12 & 10 & $\begin{array}{l}\text { Hepático-hematopoyético: } 5 \text {; } \\
\text { Renal- hematopoyético: } 7\end{array}$ \\
\hline Multisistémico: 3 sistemas & 3 & 1 & Renal-hepático-hematopoyético \\
\hline \multicolumn{4}{|c|}{$\begin{array}{l}\text { a El número se refiere a la cantidad de síndromes clínicos diagnosticados; su cantidad ( } \mathrm{n}=194 \text { con } 1 \text { complicación }+15 \text { con } 2-3 \text { complicaciones) es mayor que el número total de paciente } \\
(\mathrm{n}=126) \text { porque un enfermo puede presentar más de una complicación. El porcentaje de cada síndrome está calculado sobre el total de enfermos. }\end{array}$} \\
\hline \multicolumn{4}{|c|}{ b Otros pacientes tuvieron: GOT $>35: 17$; incluye 4 con $\mathrm{BI}>1,0$ y de esos 2 con con ALT $>40$} \\
\hline \multicolumn{4}{|c|}{ ' Otros pacientes sin las 3 alteraciones simultáneas tuvieron: a) $\mathrm{BI}>1.0+\mathrm{ALT}>40: 9$; incluye 2 con $\mathrm{BI}>1.0+\mathrm{GOT}>35 ; \mathrm{b}) \mathrm{BI}>1.0+\mathrm{GOT}>35: 4$; $\mathrm{BI}>1.0+\mathrm{ALT}>40: 2$} \\
\hline
\end{tabular}


falciparum. Los otros cuatro tenían entre 30 y 51 años de edad. La escala de Glasgow (15 puntos: consciente y orientado; $\leq 7$ indica coma) en los siete con fiebre y convulsiones varió entre cero y 15 puntos: tres casos con cero puntos (coma profundo), dos de ellos menores de cinco años y el otro con 38 años; un caso con 8 puntos; sendos casos con 8,14 y 15 puntos.

Hipoglicemia <60 mg/dL: hubo tres casos (en uno de ellos fue $<40$ ); ninguno se refirió como portador de manifestaciones neurológicas a pesar de que en dos de ellos el Glasgow se registra con cero puntos y en el tercero con 8 puntos.

La falla renal apareció en 11 pacientes: en 10 la creatinina fue $>1.2 \mathrm{mg} / \mathrm{dL}$ y en uno sucedió lo anterior junto con oliguria y anuria. La falla hepática estuvo presente en 12 enfermos, entre quienes diez mostraron únicamente alza de la bilirrubina indirecta por encima de $1.0 \mathrm{mg} / \mathrm{dL}$ y los otros dos tuvieron lo anterior más incremento de transaminasas GOT y ALT.

Anemia grave con $<7 \mathrm{~g} / \mathrm{dL}$ de hemoglobina afectó a 21 pacientes, incluidos seis con $<5 \mathrm{~g} / \mathrm{dL}$. Trece de los $21 \mathrm{ca}-$ sos tuvieron también trombocitopenia, lo que sugiere falla general hematopoyética.

Compromiso multisistémico: se halló en 15 pacientes (12\% de 126); en 12 hubo falla de dos sistemas y en tres la hubo de tres sistemas. Los comprometidos fueron siempre el renal, el hepático y el hematopoyético (anemia, trombocitopenia).

Conviene decir que 43 de los 126 pacientes fueron menores de 15 años y entre ellos hubo varios síndromes palúdicos, como trombocitopenia, anemia grave, hipoglicemia y falla hepática, y que tanto $P$. falciparum como $P$. vivax y ambas especies se hallaron presentes.

De 48 mujeres con paludismo y con 13 y más años de edad, 29 (60\%) estaban en embarazo (edad: de 14-49 años; promedio 21.8 \pm 7.2): 15 con $P$. vivax, 13 con $P$. falciparum y uno con paludismo mixto (ambas especies). La parasitemia de ellas fue de $13628 \pm 26790$ parásitos/ $\mu \mathrm{L}$ (mínimo 3 , máximo 130 000). La remisión de otra entidad sanitaria estuvo en 26 de las 29 gestantes $(90 \%)$ hospitalizadas en el HSJ. Los días de evolución de la enfermedad variaron entre 0 y 19 , con promedio de 3.9 y mediana de 3.0 días.

Tratamiento antimalárico usado: cloroquina CQ (n= $78)$, amodiaquina $\mathrm{AQ}(\mathrm{n}=2)$, quinina $\mathrm{QN}()$, artesunato AS $(n=16)$, arteméter-lumefantrina AM-LF $(n=29)$, mefloquina MQ $(n=14)$, clindamina $\mathrm{CL}(\mathrm{n}=5)$, primaquina PQ $(n=86)$. Esos medicamentos se usaron a veces como monoterapia y casi siempre combinados. La combinación más frecuente en los 76 casos de malaria por $P$. vivax fue CQ-PQ $(n=65)$, mientras que el tratamiento más común en los 39 casos de malaria por $P$. falciparum fue AM-LF (n= 21), sin asociarlo a otra droga. En 11 casos de paludismo mixto se usaron estos esquemas: CQ-PQ en cinco (en dos casos se usó también AM-LF, uno de los cuales también recibió MQ); $\mathrm{AM}-\mathrm{LF}$ en seis casos (los dos antes referidos, más otros cuatro donde fue el único esquizonticida); QN en tres casos (una como monoterapia y dos con CL+AS o con MQ+AS); AS-MQ en uno.

Los pacientes remitidos de otros lugares al HSJ fueron $93(74 \%)$ y no hubo diferencia en el tratamiento dado a los remitidos con respecto a los no remitidos, excepto en el caso de cloroquina (dada a $82 \%$ de los no remitidos vs. $55 \%$ de los remitidos; es decir, a quienes llegaron directamente al HSJ se les dio cloroquina en $82 \%$ de las veces; $\mathrm{p}=0.006$ ). Los otros medicamentos (quinina $\mathrm{p}=0.643$, artesunato $\mathrm{p}=$ 0.469 , AM-LF p $=0.212$ ) se dieron en proporciones similares a remitidos y no remitidos. La cloroquina era tratamiento de primera opción para paludismo no complicado y no parece lógico que sea el medicamento usado en pacientes que se hospitalizan, una proporción alta de los cuales tiene complicación, muy probablemente.

Mortalidad: entre los 126 pacientes, hubo cuatro defunciones (letalidad: $3 \%$ ), todas con P. vivax, en adultos (17-38 años); dos hombres y dos mujeres (un gestante); uno de los cuatro pacientes llegó remitido (gestante remitida de zona rural de Montería); los días de evolución del ataque palúdico actual variaron entre 3 y $15(3,3,5,15)$; tres con Glasgow de cero y uno con 11; tuvieron como complicación malárica éstas: a) uno falla renal únicamente; b) dos tuvieron falla de dos sistemas: uno hepática y hematopoyética (anemia grave y trombocitopenia) y uno renal y trombocitopenia; c) uno presentó falla en tres sistemas: renal, hepático, anemia grave; todos cuatro recibieron cloroquina y primaquina; sólo uno de ellos recibió quinina con clindamicina; ninguno recibió derivados de artemisinina.

\section{Discusión}

Este trabajo sobre malaria en pacientes de un hospital del tercer nivel de atención en Colombia se suma a los escasos informes sobre el tema. Técnicamente, los pacientes se hospitalizan si están graves o si tienen complicación de su enfermedad o se hallan en riesgo alto de complicarse. Sin embargo, en las zonas palúdicas colombianas sucede con frecuencia que los pacientes con paludismo se hospitalizan sin estar graves ni tener riesgo inminente de tornarse así, sino por razones sociales y operativas, como éstas: la residencia está muy distante del hospital y conviene o debe ser revisado en uno o pocos días; no hay quién cuide en la casa al enfermo; está en embarazo y debe regresar a casa a pie por varias horas; etc. Entonces, no hay que tomar "malaria en el hospital" como sinónimo de "malaria complicada", porque en esas zonas endémicas colombianas no es así.

Hemos venido avanzando en la formulación de propuestas tendientes a definir unos criterios autóctonos sobre paludismo grave y sus diversos síndromes. En 2000 con base en un estudio sobre paludismo por $P$. vivax o $P$. falciparum en pacientes internados en cinco grandes hospitales de Medellín, presentamos una propuesta, que se aparta de la formulada por OMS en varios puntos (5). En el presente trabajo, retomamos los criterios de González et al 2000 (5) 
y les hicimos leves modificaciones. En 2006, en dos escritos (24-25), enunciamos los criterios usados para evaluar pacientes con malaria grave por P. falciparum y los confrontamos con los criterios de OMS, resaltando las diferencias.

Un aporte interesante de este trabajo es poder mirar cómo se tratan ahora, en la práctica rutinaria, los pacientes palúdicos hospitalizados, concretamente en un hospital de tercer nivel. Junto con un informe sobre malaria en pacientes del hospital de segundo nivel en Apartadó (Urabá antioqueño) (3) y otros sobre paludismo complicado en pacientes de los hospitales Tumaco (Costa Pacífica en el departamento de Nariño) y Turbo (Urabá antioqueño) (24-25), servirán de manera notoria para formarse una idea de lo que sucede ahora en el país en esas entidades en cuanto al tratamiento palúdico se refiere. Para nosotros el panorama no es técnicamente adecuado.

La especie plasmodial informada con la gota gruesa realizada en el HSJ corresponde, en general, a lo conocido: predominio de alrededor de $60-70 \%$ de $P$. vivax, pero la frecuencia de paludismo mixto si es alta $(9 \%)$ con respecto a lo visto en la población general de casos de esta región, que es de $1.44 \%$ (26-27). Se dice que las coinfecciones plasmodiales son mutuamente supresivas, con P.falciparum dominando a $P$. vivax y esté atenuando la gravedad de la infección por $P$. falciparum y que no hay bases suficientes para orientar el tratamiento de la infección plasmodial y malaria mixta, pero se sugiere que debe darse tratamiento simultáneo para las diferentes especies (26-27).

La parasitemia promedio vista en estos enfermos (13 628 \pm 26 790) es el doble de lo apreciado en los pacientes ambulatorios no complicados en esta región: promedio de 8766 parásitos/ $\mu \mathrm{L}$ y mediana de 5400/ $\mu \mathrm{L}$ (28). Sólo uno de cada diez pacientes hospitalizados en Montería mostró >50 000 parásitos/ $\mu \mathrm{L}$, valor por encima del cual se considera en Colombia que existe malaria grave.

El tiempo promedio de evolución del ataque actual de paludismo, que motivó la hospitalización (4-5 días) es similar al narrado por pacientes ambulatorios que reciben tratamiento antipalúdico en la misma región: cuatro días como mediana y 5.8 como promedio (28). En Apartadó (Urabá antioqueño) en pacientes de un hospital regional de grado 2 de complejidad de atención, durante 2004-2007, se encontró un promedio de evolución de los síntomas de 5.6 \pm 5.8 días (1 y 60 días) (3).

El valor promedio de los signos vitales se halló en el intervalo fisiológico; eso, junto con que el promedio de la hemoglobina indica anemia moderada y que, en general, los leucocitos tienen cantidad y distribución relativa normal, y los promedios de glicemia, creatinina y BUN son normales, indica que el deterioro de los pacientes no era alto, aunque los promedios de urea y bilirrubinas fueron altos. Entonces, si el cuadro clínico no era grave, llama la atención que tres de cada cuatro pacientes llegasen al HSJ remitidos de otros lugares. ¿Esto hablará de deficientes condiciones de atención a los pacientes, bien sea por la capacidad del personal, de las dotaciones técnicas y de medicamentos u otras razones? Una parte importante de la explicación tiene que ver con la población de mujeres en embarazo y con paludismo, pues $90 \%$ de ellas llegaron al HSJ remitidas de otros municipios. Parece un asunto importante para evaluar desde el punto de vista de la planificación y administración de servicios de salud. En esa región, las malarias gestacional y placentaria son problema de salud pública por su elevada frecuencia y los efectos que producen en madres e hijos $(15,29-35)$.

Es muy importante señalar que uno de cada tres pacientes hospitalizados $(34 \%=43 / 126)$ fueron menores de 15 años. Una revisión de 2011 sobre malaria grave en América Latina mostró que en la práctica no hay datos ni en adultos ni en niños y que es urgente adelantar estudios autóctonos, que incluyan las diferentes formas clínicas causadas por $P$. falciparum y $P$. vivax (36).

Los pacientes con evaluación "completa" de laboratorio fueron muy pocos y esto no es aceptable, pues son enfermos que por estar en un hospital de nivel 3, por haber sido en su mayoría remitidos allí y por sospecharse que tiene paludismo complicado, debieran tener resultados de una batería amplia de exámenes para poder precisar un diagnóstico. ¿Cómo lograr diagnósticos oportunos y correctos y poder aplicar los tratamientos adecuados si los enfermos hospitalizados no se someten a evaluaciones paraclínicas rigurosas y rutinarias de elevada calidad?

La frecuencia de síndromes clínicos diagnosticados se asemeja sólo en parte a la hallada en el hospital regional de Apartadó, hacia 2012 (3). En este sitio no hallaron complicación cerebral que en el HSJ fue $6 \%$ (ocho casos: cinco por $P$. vivax, dos por P. falciparum, uno mixto). El estudio de Apartadó no define directamente qué entendió por malaria cerebral, pero dice que se ajustó al criterio de OMS en 2000, el cual la define como la presencia de coma según las escalas de Glasgow $<9$ o Blantyre $<2$. Esto es muy diferente a lo aplicado a los pacientes del HSJ, para los cuales se habló de "compromiso cerebral" definido como el paciente que "tiene simultáneamente fiebre y convulsiones, o fiebre y coma, o fiebre, convulsiones y coma. Con los tres criterios actuando en forma concomitante el diagnóstico de paludismo cerebral parece altamente probable, haciéndose casi seguro si la especie parasitaria asociada es $P$. falciparum o éste y $P$. vivax en forma simultánea (malaria mixta)". Sólo un paciente mostró Glasgow $<9$ (tuvo 8 puntos), tuvo fiebre y convulsiones pero no coma; tenía $P$. falciparum. En un estudio de malaria grave en Tumaco y Apartadó, hacia 2006 (24-25), se encontró una frecuencia de malaria cerebral de $3 \%$ con el mismo criterio de Arboleda y colaboradores.

Enorme diferencia hubo también en la hemoglobina $<7$ $\mathrm{g} / \mathrm{dL}$, que en Apartadó fue de $61 \%$ contra sólo $17 \%$ en Montería. No tenemos explicación para esta discrepancia, porque ambas mediciones se aplicaron a cianometahemoglobina y usaron equipos automatizados "modernos". 
Otros síndromes no tuvieron tanta diferencia como los dos anteriores (Montería vs. Apartadó): alteración renal con creatinina $>1.5 \mathrm{mg} / \mathrm{dL}$ en $4 \%$ contra $3 \%$; disfunción hepática con bilirrubina total $>3$ en $12 \%$ contra $8 \%$; incremento de enzima ALT sérica en $0 \%$ contra $4 \%$; recuento plaquetario $<20000$ en $6 \%$ frente a 18\%; proporción de falla multisistémica fue $11 \%$ contra $12 \%$.

Los tratamientos aplicados en el HSJ llaman la atención porque fueron en general los recomendados para paludismo no complicado (pacientes ambulatorios), es decir, tratamiento oral con cloroquina-primaquina para $P$. vivax y arteméter-lumefantrina en casos debidos a $P$. falciparum (37). No es fácil entender que a pacientes internados en un hospital de nivel tres, se les dé tratamiento oral como si estuviesen ambulatorios, en vez de usar medicamentos por vía intramuscular o intravenosa (37).

Los pacientes remitidos de otros lugares fueron $74 \%$ y no hubo diferencia en el tratamiento dado a ellos con respecto a los no remitidos, excepto en la cloroquina (dada a $82 \%$ de no remitidos vs. $55 \%$ de remitidos). Cabe preguntar cuál fue la diferencia en el tratamiento tras la remisión, o para qué sirvió la remisión según los medicamentos antipalúdicos ofrecidos.

Las cuatro defunciones representan más de $3 \%$ del total de 126 enfermos. La mortalidad palúdica ha tenido marcada tendencia descendente en el país, registrándose, según el DANE, un promedio de 130-150 muertes/año en 2001-2010 (38) o de 65-165 muertes/año en 1999-2009 (39). Existe subregistro mayor de $60 \%$ en la mortalidad por paludismo, principalmente en la costa Pacífica (38). Otro informe reciente sobre mortalidad palúdica en Colombia entre 1979 y 2008 (30 años), dice que se certificaron 6965 muertes por paludismo (232 muertes/año); que en $74.3 \%$ de las defunciones no se especificó la especie parasitaria y que entre 1790 defunciones con especie identificada se halló $P$. falciparum en $21 \%, P$. vivax en $4 \%, P$. malariae en $0.2 \%$, y otra especie parasitaria en $0.5 \%$ ("incluida las especies asociadas y $P$. ovale” sic) (40).

Concluimos que:

1. Es obvia la importancia de $P$. vivax en la enfermedad que afecta a estos pacientes, pues no sólo es mayoría como causa de ella sino que fue la especie responsable de las cuatro defunciones. Esto debe reforzar en Colombia la alerta creciente en el mundo sobre la gravedad del paludismo vivax.

2. Los hallazgos sobre complicaciones con criterios más flexibles que los de OMS 2000 reiteran la conveniencia de avanzar en la construcción de normas generales de consenso colombiano sobre el tema, pues al parecer los criterios de OMS basados en la experiencia africana y en $P$. falciparum no se ajustan adecuadamente a la realidad colombiana, según lo indican las investigaciones ejecutadas en el país sobre paludismo grave.

3. Es alarmante que $60 \%$ de los casos de paludismo hospitalizados en el HSJ correspondan a gestantes. Esto pone de manifiesto otra vez lo que recientemente se ha empezado a conocer en el país: la malaria gestacional es un grave problema de salud pública y debe recibir con urgencia toda la atención para que las mujeres y sus hijos puedan ser atendidas como se requiere.

4. Es francamente incompleta la evaluación de los pacientes con pruebas básicas de laboratorio clínico, porque, con excepción del hemograma, exámenes elementales como transaminasas, creatinina, urea, bilirrubinas y glicemia sólo se hicieron a $25-48 \%$ de los enfermos, lo cual pone de manifiesto problemas graves en la atención.

5. Al parecer hay inadecuado uso de la terapia antimalárica para pacientes hospitalizados, pues con muy elevada frecuencia recibieron el mismo tratamiento, por la misma vía y las mismas dosificaciones que el que se aplica a pacientes ambulatorios no complicados, a pesar de que estos pacientes en realidad tenían mucho más que paludismo no complicado.

\section{Financiación}

Esta investigación fue financiada por: a) Colciencias, con fondos de los proyectos 111549326134 (Contrato $N^{\circ} 611$ de 2009) y 111556933361 (444-2013); b) Banco de la República (3069; IMM 8764-2555 Acta E0 1717); c) Estrategia de Sostenibilidad Codi-Universidad de Antioquia 2014-2015; d) Universidad de Antioquia.

Ninguno para manifestar.

\section{Declaración de conflictos}

\section{Referencias}

1. WHO. World Health Organization, The Royal Society of Tropical Medicine and Hygiene. Severe falciparum malaria. Trans Roy Soc Trop Med Hyg. 2000; 94 (Supl 1): 1-10.

2. Alexandre M, Ferreira C, Siqueira A, Magalhães B, Mourão M, Lacerda M, et al. Severe Plasmodium vivax Malaria, Brazilian Amazon. Emerg Infect Dis. 2010; 16 (10): 1611-4.

3. Arboleda M, Pérez MF, Fernández D, Usuga LY, Meza M. Perfil clínico y de laboratorio de los pacientes con malaria por Plasmodium vivax, hospitalizados en Apartadó, Colombia. Biomédica. 2012; 32 (supl.1): 58-67.

4. Barbara S. Focus on Plasmodium vivax. Trends Parasitol. 2002; 18 (7): 287-9.

5. González L, Guzmán M, Carmona J, Lopera T, Blair S. Características clinico-epidemiologicas de 291 pacientes hospitalizados por malaria en Medellin (Colombia). Acta méd Colomb. 2000; 25 (4): 163-70.

6. Kochar D, Das A, Kochar S, Saxena V, Sirohi P, Garg S, et al. Severe Plasmodium vivax malaria: A report on serial cases from Bikaner in Northwestern India. Am J Trop Med Hyg. 2009; 80 (2): 194-8.

7. Lacerda MV, Hipólito GR, Passos LNM. Chronic Plasmodium vivax infection in a patient with splenomegaly and severe thrombocytopenia. Rev Soc Bras Med Trop 2008; 41 (5): 522-523. 2008; 41 (5): 522-3.

8. Lacerda MVG, Mourao PPG, Alexandre MAA, Siqueira AM, Magalhaes BML, Martinez-Espinosa FE. Understanding the clinical spectrum of complicated Plasmodium vivax malaria: a systematic review on the contributions of the Brazilian literature. Malar J. 2012; 11: 12.

9. Picot S. Plasmodium vivax still a paradigm for uncomplicated malaria?. Med Mal Infect. 2006; 36 (6): 406-13.

10. WHO. World Health Organization Action Programme. Severe and Complicated Malaria. Trans Royal Soc Trop Med Hyg. 1986; 80 (supplement): 3-50.

11. WHO. World Health Organization, Division of Control of Tropical Diseases. Severe and Complicated Malaria. Trans Royal Society Trop Med Hyg. 1990; 84 (supplement 2): 1-65.

12. WHO. World Health Organization. Guidelines for the treatment of malaria. WHO/ HTM/MAL/2006. 1108. Geneva: WHO; 2006.

13. Campos FMF. Marcadores de inflamação na morbidade da malária por Plasmodium vivax: contribuição de micropartículas, ácidos nucléicos circulantes e polimorfismos genéticos do hospedeiro vertebrado. Belo Horizonte; s.n; 2011. xviii,118 p. Portugués. Tese: Apresentada a Centro de Pesquisas René Rachou para obtenção do grau de Doutor 2011. 
14. Maestre A, Carmona-Fonseca J. Immune responses during gestational malaria: a review of the current knowledge and future trend of research. J Infect Dev Ctries. 2014; 8 (4): 391-402.

15. Yasnot M,F.,Perkins JD, Corredor M, Yanow S, Carmona-Fonseca J, Maestre A. Efectos de la malaria gestacional por Plasmodium vivax sobre el estado clínico e inmune en gestantes del Noroccidente de Colombia. Colomb Méd. 2013; 44 (3): 172-7.

16. Espinosa FE. Malária na gravidez: estudo de pacientes do Instituto de Medicina Tropical do Amazonas, Brasil, 1990-1997. Rio de Janeiro; s.n; 1998.142 p. tab. Apresentada a Instituto Oswaldo Cruz para obtenção do grau de Mestre. 1998.

17. Carvajal C, García L, Gottberg E, González C, Longa I, Pires M. Incidencia de malaria cerebral en el Hospital Pediátrico "Menca de Leoni”. San Félix estado Bolívar: periodo 1993-2004. Arch venez pueric Pediatr. 2006; 69 (3): 103-7.

18. Colombia. , Proyecto Malaria Colombia (PMC). Reporte de la situación de malaria en los 45 municipios priorizados del Proyecto Malaria Colombia. Períodos epidemiológicos 1 a 6 de 2013. Consulta: 10 octubre 2014. http://www.proyectomalariacolombia.co/files/InfEpiConsolidadoP1a6_2013.pdf. 2013.

19. Morales-Romero OC, Morales-Romero YE. Caracterización del comportamiento de la malaria en del departameto de Córdoba durante el periodo 2001-2007. Tesis de grado, especilización en Gerencia en Salud Pública, Universidad CES, Medellín, 2009. 40 p.

20. Carmona-Fonseca J. La malaria en Colombia, Antioquia y las zonas de Urabá y Bajo Cauca: panorama para interpretar la falla terapéutica antimalárica. Parte 1. Iatreia. 2003; 16 (4): 299-318.

21. Carmona-Fonseca J. La malaria en Colombia, Antioquia y las zonas de Urabá y Bajo Cauca: panorama para interpretar la falla terapéutica antimalárica. Parte 2. Iatreia. 2004; 17 (1): 34-53.

22. Padilla JC, Álvarez G, Montoya R, Chaparro P, Herrera S. Epidemiology and control of malaria in Colombia. Mem Inst Oswaldo Cruz, Rio de Janeiro. 2011; 106 (Suppl. I): 114-22

23. Sánchez López YL, Carmona-Fonseca J. Contexto del paludismo en la región Urabá Antioqueño-Altos Sinú y San Jorge-Bajo Cauca Antioqueño. Informe de investigación (tesis). Grupo Salud y Comunidad-César Uribe Piedrahita, Universidad de Antioquia; Medellín, diciembre 2014.

24. Tobón A, Giraldo C, Pineros J, Arboleda M, Blair S, Carmona-Fonseca J. Epidemiología de la malaria falciparum complicada: estudio de casos y controles en Tumaco y Turbo, Colombia, 2003. Rev bras epidemiol. 2006-b; 9 (3): 283-96.

25. Tobón A, Piñeros J, Blair S, Carmona-Fonseca J. Clínica de la malaria complicada debida a $P$. falciparum Estudio de casos y controles en Tumaco y Turbo (Colombia). Iatreia. 2006-a; 19 (4): 339-55.
26. Carmona-Fonseca J, Arango E. Malaria mixta: prevalencia en Colombia y América Latina. Iatreia. 2012-a; 25 (4): 334-46.

27. Carmona-Fonseca J,Arango E. Malaria mixta: revisión general. Méduis. 2012-b; 25 (1): $45-54$

28. Carmona-Fonseca J, Tobón A, Álvarez G, Blair S. El tratamiento amodiaquinasulfadoxina-pirimetamina tiene eficacia del $98 \%$ para la malaria falciparum no complicada (Antioquia, Colombia; 2003). Iatreia. 2005; 18 (1): 5-26.

29. Agudelo O, Arango E, Maestre A, Carmona-Fonseca J. Prevalence of gestational, placental and congenital malaria in north-west Colombia. Malar J. 2013; 12: (341).

30. Agudelo OM, Aristizabal BH, Yanow SK, Arango E, Carmona-Fonseca J, Maestre A. Submicroscopic infection of placenta by Plasmodium produces Th1/ Th2 cytokine imbalance, inflammation and hypoxia in women from north-west Colombia. Malar J. 2014; 13: (122).

31. Arango E, Maestre A, Carmona-Fonseca J. Effect of submicroscopic or polyclonal Plasmodium falciparum infection on mother and gestation product: systematic review. Rev Bras Epidemiol. 2010; 13 (3): 373-86.

32. Arango EM, Samuel R, Agudelo OM, Carmona-Fonseca J, Maestre A, Yanow SK. Molecular detection of malaria at delivery reveals a high frequency of submicroscopic infections and associated placental damage in pregnant women from Northwest Colombia. Am J Trop Med Hyg. 2013; 89 (1): 178-83.

33. Campos IM, Uribe ML, Cuesta C, Franco-Gallego A, Carmona-Fonseca J, Maestre A. Diagnosis of gestational, congenital, and placental malaria in Colombia: comparison of the efficacy of microscopy, nested polymerase chain reaction, and histopathology. Am J TropMed Hyg. 2011; 84 (6): 929-35.

34. Carmona-Fonseca J, Maestre A. Incidencia de las malarias gestacional, congénita y placentaria en Urabá (Antioquia, Colombia) 2005-2007. Rev Col Obst Ginecol. 2009; 60 (1): 19-33.

35. Carmona-Fonseca J, Arias MM, Correa A, Lemos M. Malaria gestacional y condiciones de vida. Medicina Social. 2011; 6 (2): 97-107.

36. Carmona-Fonseca J. Malaria grave en niños. Méduis. 2011-c; 24 (2): 181-94.

37. Colombia. , Ministerio de la Protección Social. Guía para la atención clínica integral del paciente con malaria. Bogotá: Ministerio de la Protección Social, 2010. 2010 .

38. Colombia. Instituto Nacional de Salud (INS), Subdirección de Vigilancia y Control en Salud Pública. Protocolo de vigilancia y control de malaria. Bogotá: INS. Agosto de 2011. 2011.

39. Padilla JC, Montoya R. Guía de Atención Clínica de Malaria. Infectio. 2011; 15 (4): 302-23.

40. Chaparro P, Padilla J. Mortalidad por paludismo en Colombia, 1979-2008. Biomédica 2012; 32 (Supl.): 95-105. 